\section{NOVA TELLVS}

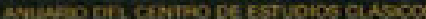

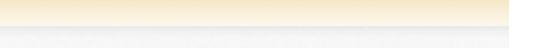

Nova Tellus

ISSN: 0185-3058

novatelu@servidor.unam.mx

Centro de Estudios Clásicos

México

Schmidt Osmanczik, Ute

Aspectos lúdicos en el Protágoras de Platón y unas cuantas contradicciones

Nova Tellus, vol. 26, núm. 2, 2008, pp. 121-133

Centro de Estudios Clásicos

Distrito Federal, México

Disponible en: http://www.redalyc.org/articulo.oa?id=59115497005

Cómo citar el artículo

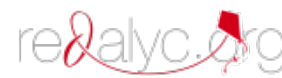

- Número completo

- Más información del artículo

Página de la revista en redalyc.org

Sistema de Información Científica

Red de Revistas Científicas de América Latina, el Caribe, España y Portugal

Proyecto académico sin fines de lucro, desarrollado bajo la iniciativa de acceso abierto 


\title{
Aspectos lúdicos en el Protágoras de Platón y unas cuantas contradicciones
}

\author{
Ute SCHMIDT OSMANCZIK \\ Universidad Nacional Autónoma de México \\ alster12@prodigy.net.mx
}

RESUMEN: En su diálogo Protágoras, Platón hace derroche de buen humor en varios pasajes, rasgo que se contrapone a su habitual seriedad. En este pequeño escrito quiero rescatar algunos de estos pasajes a la vez que deseo mostrar algunas contradicciones y errores.

$$
* * *
$$

ABSTRACT: In his dialogue Protagoras, Plato shows in various passages a very good sense of humour, which is not usual in him. In this little article I want to point out some of these passages, and, at the same time, I wish to exhibit some contradictions and errors.

Palabras Clave: aspectos lúdicos, ética, filosofía griega, Platón, Protágoras. RECEPCIÓN: 14 de agosto de 2008.

ACEPTACIÓN: 13 de octubre de 2008. 


\title{
Aspectos lúdicos en el Protágoras de Platón y unas cuantas contradicciones
}

\author{
Ute SCHMIDT OsMANCZIK
}

La manera platónica de escribir en forma de diálogo, no de tratado, permite la ficción de conversaciones. Dichas conversaciones, que en el Platón viejo se asemejan muchas veces a tratados, se desarrollan en determinado "ambiente" que puede ser de extrema seriedad y tristeza (por ejemplo en Fedón), puede presentar un tono estrictamente científico (por ejemplo en Teeteto) o ser de otra índole. Por lo general, Platón se decide por un tono más bien serio; pocos son los lugares en los cuales el lector sonríe o francamente ríe durante la lectura.

En el Protágoras participan muchos interlocutores; esta obra es un diálogo que realmente ofrece, en varias partes, un derroche de buen humor, aspecto rarísimo en la obra platónica (con la excepción del Eutidemo). En este pequeño artículo quisiera rescatar unos cuantos de estos aspectos, junto con algunos otros puntos relevantes.

Desde hace varios años estoy impartiendo el curso "Lectura de textos filosóficos griegos". En dos ocasiones opté por leer, junto con los alumnos, el diálogo platónico Protágoras (cuyo contenido doy aquí por sabido), y fue en estas ocasiones en las que me percaté de que esta obra resulta temática y estilísticamente abierta; tiene de todo un poco: pasajes muy serios e importantes, como, por ejemplo, el tema de la enseñanza y procedencia de la virtud, la unidad de las virtudes y la asimilación de aspectos morales al modelo de la téchne; pero también se dan situaciones realmente cómicas y/o irónicas y no faltan contradicciones y falsedades, todo lo cual constituye

NOVA TELLVS, 26•2, 2008 
el tema de este escrito. Hay que añadir que en el diálogo que lleva su nombre, Protágoras, el sofista, se muestra, en términos generales, bastante más sensato que Sócrates, asunto al que también me voy a referir, si bien ello no figura en el título de este pequeño ensayo.

El trabajo aquí presentado es fruto de enseñanza, mucho más que de investigación. Los puntos que mencionaré a continuación no me saltaron a la vista cuando traduje la obra de la que aquí se trata; llamaron mi atención al leer el diálogo en clase con los papeles repartidos entre los alumnos, cuando yo misma me convertí en buena medida en un "espectador" u "oyente" de esta obra muy vivaracha; era como si la leyera por primera vez. Tal es la razón por la cual este trabajo carece de una bibliografía explícita.

Este artículo presenta tres apartados: en primer lugar, se refiere a lugares cómicos y/o irónicos; en segundo lugar, a ciertas contradicciones y en tercer lugar, a la sensatez que Protágoras exhibe en la obra que Platón le dedica.

\section{Lugares cómicos e irónicos}

En primer lugar, el Protágoras empieza con unas palabras que hacen reír o sonreír de inmediato: se alude a una situación homoerótica, de la cual todo platonista sabe que no era tal. Dice uno de los interlocutores de la obra: “¿De dónde vienes, Sócrates? ¿No es obvio que de rondar la floreciente edad de Alcibíades?"1 Y en efecto, Sócrates confirma que justo viene de ver a Alcibíades, el bello Alcibíades, pero que vio a alguien aún más bello, a saber, al sabio Protágoras. En cuanto a Alcibíades, sabemos cómo era su relación con Sócrates en realidad: no fue de modo alguno homoerótica, pues como se

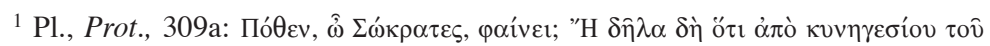

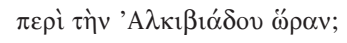


sabe bien por el final del Banquete, Sócrates había rechazado tajantemente sus insinuaciones eróticas; pese a ello, Sócrates no se indigna acerca de la alusión de buscar a Alcibíades, sino que se sigue prestando a las habladurías de tener que ver algo con el joven.

En el Protágoras se presenta una situación que se suele llamar el "congreso de los sofistas", porque en la escenografía del diálogo se reúnen muchos de ellos (cf. 314d), como Hipias de Elis y Pródico de Ceos. El segundo punto que se refiere a una situación cómica tiene que ver con este "congreso", en el cual se manifiesta una deliciosa e irónica rivalidad por un lado entre Protágoras, que encanta, como Orfeo, a sus seguidores (cf. 315b) y Pródico e Hipias, por otro. Señala Sócrates que él sospechaba que Protágoras "quería darse importancia y mostrar a Pródico e Hipias” que Sócrates e Hipócrates, un probable alumno de Protágoras, habían llegado como "admiradores" de Protágoras. Se quiere dar a entender que no habían llegado como admiradores de los otros dos sofistas. Más adelante sigue el juego; dice Protágoras: "Ahora, Sócrates, ya que éstos [Hipias y Pródico] están presentes, podrías decirme lo que poco antes mencionabas acerca de este joven" [Hipócrates]. ${ }^{2}$ Protágoras se exhibe delante de Pródico e Hipias; quiere dar la impresión de ser más competente que ellos, y da rienda suelta a cierta vanidad.

Dentro de la misma rivalidad (cf. 318 d-e), Protágoras "ataca" a otros sofistas por el tipo de enseñanza que imparten; hablando de Hipócrates, el alumno en ciernes, dice:

...si llega conmigo, no sufriría lo que hubiera sufrido al juntarse con algún otro sofista, pues los otros maltratan a los jóvenes, a quienes, ya libres de las artes, conducen en contra de su voluntad de nueva cuenta a las artes, enseñándoles cálculo, astronomía, geometría y música $-y$ en eso miró a Hipias -; pero si llega

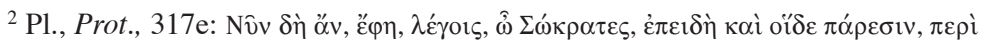

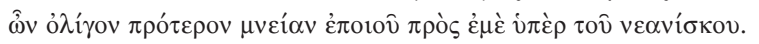


conmigo, no aprenderá otra cosa, sino aquella por la cual vino. (Subrayados míos.)

En otras palabras, Hipias maltrata a sus alumnos con algo tan común como materias de tipo escolar, mientras que él, Protágoras, les enseña algo de más valía, a saber, ocuparse exitosamente de los asuntos de la ciudad, administrar bien sus casas, ser buenos ciudadanos y oradores.

En tercer lugar, Sócrates a su vez "ataca" a Protágoras amablemente unas cuantas veces: (a) cuando (cf. 328d) Protágoras terminó un discurso largo acerca de la posiblilidad de enseñar la virtud y acerca de su propia utilidad como maestro, Sócrates comenta: "yo, todavía fascinado, lo contemplaba por mucho tiempo..." (subrayados míos); pensaba que Protágoras iba a decir todavía algo verdaderamente extraordinario y fuera de lo común. (b) Más adelante (cf. 328e), Sócrates manifiesta que tiene una duda pequeña - que en realidad es grande - en torno al tema de si la virtud es una o no, pero que no se preocupa por ello, pues está seguro de que Protágoras obviamente resolverá el asunto con suma facilidad, lo que por cierto no fue el caso. (c) En determinado momento de la conversación se presenta una situación en la que se quiere buscar un "conductor" del debate. ¿Quién será? Anuncia Sócrates que claramente este papel le corresponde a Protágoras, pues él es el más sabio (cf. 338c).

En cuarto lugar, el lector sonríe con las distinciones semánticas de Pródico, y en esta ocasión el sofista se luce bastante. "Imparcial" no es lo mismo que "igual"; se debe "discutir", mas no "disputar", se puede ser "estimado", pero no "alabado" y se tiene "recocijo", pero no "placer" (cf. 337a ss.). Luego se hace la distinción entre "querer" y "desear" (cf. 340b).

En quinto lugar, en el curso de la obra, llega un momento en que Sócrates y Protágoras están de acuerdo en que el ser capaz de hablar de poesía forma parte de la cultura de una persona. En este contexto se trae a colación una oda de Simó- 
nides, la cual se pretende interpretar. La exégesis del poema de Simónides presenta un tratamiento irónico de cierto tipo de discurso; es una brillante parodia de una lectura sofística. Sin lugar a dudas, la conversación que se da en torno a esta oda es uno de los lugares del Protágoras que más incitan a la risa; hay derroche de gracia y de sofismas intencionados por parte de Platón/Sócrates. Valdría la pena ver todo el pasaje, pero por razones de espacio me tendré que restringir a unos pocos puntos. Pero antes de ello, es lícito preguntarse si todo este derroche de sofismas tiene un fondo serio, porque el asombrado lector se plantearía ¿esto es lo que iban a aprender los futuros alumnos de Protágoras?

La discusión sobre el poema de Simónides es muy larga y compleja; la oda trata, al menos parcialmente, el tema de la virtud. En ella Simónides afirma que "en verdad, difícil es por cierto devenir un hombre bueno, sólido de manos, pies y espíritu, hecho sin falta". ${ }^{3}$ Más adelante se lee: "no me es suficiente medida la palabra de Pítaco, aunque dicha por un hombre sabio: difícil es permanecer bueno". "Una vez que Protágoras concluye su comentario acerca del texto, Sócrates se muestra "asustado" ante la elocuencia del sofista y dice: “...en un primer momento, como golpeado por un buen pugilista [que sería Protágoras] se me nubló la vista y me dio un mareo..." (subrayado mío). ${ }^{5}$

Durante la discusión en torno al poema, hay una interpretación de Sócrates donde sostiene que si bien es difícil llegar a ser un hombre bueno, una vez siéndolo, es fácil permanecer en el estado de bondad (cf. 340d), lo cual es difícil de aceptar y sumamente rebuscado, y además exactamente el contrario

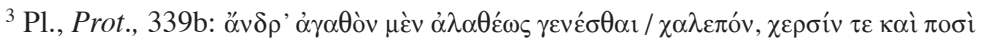

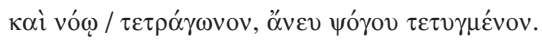

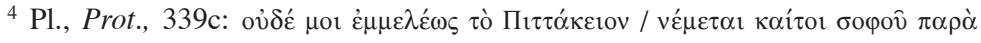

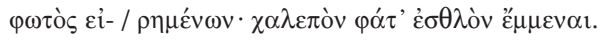

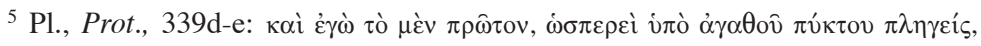

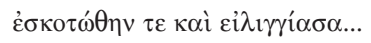


de lo que se acaba de indicar líneas arriba. Protágoras muestra gran sensatez al sostener lo siguiente: "sería gran ignorancia del poeta ... si afirmara que poseer la virtud es algo simple, lo cual es lo más difícil de todo, como les parece a todos los hombres" (340 e).

Luego, un poco más adelante, se presenta un punto verdaderamente grotesco, si bien se señala que puede tratarse de una broma: Sócrates / Platón hace que Pródico, el sofista famoso por sus conocimientos acerca del significado de las palabras, interprete en determinado momento el vocablo "difícil"

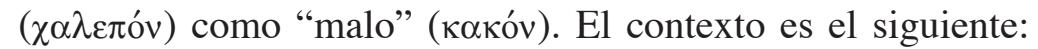
'es difícil permanecer bueno', esto es, es difícil mantener el estado de bondad. Ahora bien, si "difícil" significa "malo", tendríamos la lectura fantástica: "es malo permanecer bueno"

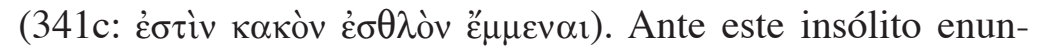
ciado, Protágoras actúa con sensatez y contesta: "Yo sé bien que... Simónides se refería con 'difícil', como lo hacemos todos nosotros, no a lo 'malo', sino a 'lo que no es fácil... lo que se obtiene con mucho trabajo" (341d).

Hay otro lugar extremadamente curioso en la misma oda de Simónides. Al principio del poema se había dicho "en verdad ( $\dot{\alpha} \lambda \alpha \theta \dot{\varepsilon} \omega \varsigma)$, difícil es... devenir un hombre bueno ...” (cf. 339b). En 343d se retoma el mismo asunto: “...devenir un hombre bueno es... difícil de verdad...", ${ }^{6}$ y se añade, un poco incomprensiblemente "no uno bueno en verdad ...". Sócrates señala que se tiene que interpretar del modo siguiente: "de verdad" no se dice en el sentido "...como si hubiera algunos que son buenos de verdad y otros que son buenos, pero no de verdad..." (cf 343e). El "en verdad" simplemente se debe leer como un hipérbaton. Aquí, y también en lo que sigue (cf. 343 ss.), Platón hace gala de una erudición sofística que cualquier sofista le envidiaría.

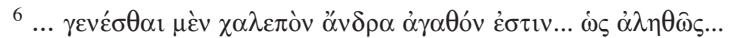




\section{Contradicciones}

En primer lugar, Protágoras opina que la virtud es enseñable, que su propia enseñanza es "la prudencia en asuntos familiares... y en los asuntos de la ciudad..." (318e-319a). Para demostrar su tesis a Sócrates, relata un mito, una fábula, la cual termina por afirmar que la virtud (política) es un don divino que fue dado a todos los hombres, esto es, a cada individuo; ello equivale a sostener que es innata, tesis que posteriormente se desarrollará detenidamente en el Menón. Ahora bien, si todos tenemos la virtud por intervención divina, no hace falta que se enseñe. Sin embargo, en lo que sigue, Protágoras se afana por aducir argumentos que pretenden demostrar la enseñabilidad de la virtud (cf. 323a ss.). El sofista demuestra, pues, justamente lo contrario de lo que quería demostrar.

En segundo lugar, el tema de los discursos largos y cortos es otro punto que muestra contradicciones. En 334d, Sócrates anuncia que es olvidadizo, enunciado del que cualquier conocedor de Sócrates se ríe, aparte de saber que es completamente falso, y que Alcibíades rectificará un poco más adelante, y por ello le pide a Protágoras: "recórtame las respuestas".7 Pues bien, a la vez que pide a Protágoras que conteste con brevedad, el mismo Sócrates lanza después discursos bastante largos: uno de 16 líneas (cf. 335d-336b), otro de 25 líneas (cf. 338 b-e) y otro más que comprende desde $342 \mathrm{a}-347 \mathrm{a}$.

En tercer lugar, quiero hacer hincapié en una simple falsedad. En 345a leemos lo siguiente: “¿Quién podría devenir un médico malo? Obviamente quien primero resulta médico y luego, un médico bueno, pues éste podría devenir también

\footnotetext{
${ }^{7}$ Esto recuerda una situación en el Gorgias (cf. 449d), donde el propio Gorgias, exhortado por Sócrates para que responda brevemente, se contentó en dos ocasiones consecutivas con un simple "sí", a lo que su interlocutor dijo: "por Hera, me gustan tus respuestas".
} 
malo", y más adelante ${ }^{8}$ siempre en relación con el médico: un médico sólo puede llegar a ser malo si antes era bueno. Ahora bien, esto no es en modo alguno así pues se puede ser un mal médico desde un principio; no hace falta haber sido bueno al principio de una carrera para luego llegar a ser incompetente. Así, Platón aplica el mismo razonamiento a la calidad moral de una persona: alguien puede llegar a ser moralmente malo sólo si antes era bueno, lo cual también es falso: se puede ser malo desde un principio.

\section{La sensatez de Protágoras}

Por último, quisiera mostrar algunos pasajes en los que se manifiesta una marcada sensatez de Protágoras, lo cual no se puede afirmar de Sócrates en estos mismos pasajes.

El primer punto a abordar en este contexto es el de la unidad de las virtudes. En el diálogo se presenta la siguiente situación: Sócrates quiere demostrar a toda costa que la justicia, la moderación, la sabiduría, sophía (que también se puede traducir por "inteligencia"), la piedad y la valentía son una sola virtud. Este tema ocupa muchas páginas de la obra y, en un primer momento, Protágoras señala muy correctamente: "Muchos son valientes, pero injustos, y muchos a su vez son justos, pero no sabios" (329e). Más adelante, el mismo Protágoras admite que la piedad, la justicia, la moderación y la sabiduría se parecen en demasía (cf. 349d), pero “...la valentía es mucho muy distinta de todas éstas. Y de la siguiente manera reconocerás que digo la verdad: encontrarás muchos hombres que son extremadamente impíos, desenfrenados e ignorantes, pero valientes en grado excelso". 9

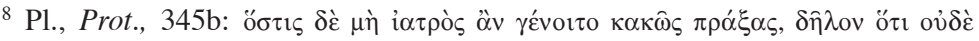

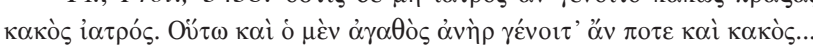

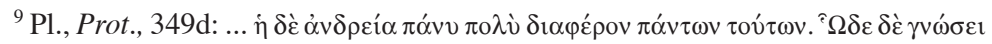


Esto es indudablemente cierto, pero Sócrates es tenaz. Nuevamente en relación con la valentía, Protágoras critica un modo de argumentar socrático, mostrando un gran sentido común. Se argumenta más o menos así (cf. 349e ss.): los valientes son, aparte de valientes, temerarios, y se arriesgan a muchas cosas a las que la mayoría de las personas no se arriesgaría. Ahora bien, por otro lado, la valentía es parte de la virtud, y ésta es en su conjunto algo bello. Las personas que temerariamente se lanzan al agua, lo hacen porque saben nadar, esto es, tienen el conocimiento de la natación, son expertos. Los conocedores

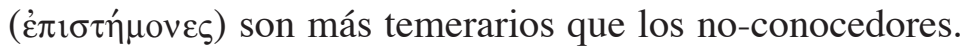

Pero, también hay individuos que sin conocimiento de la natación se arrojan al agua. En tal caso la valentía se convertiría en algo feo, y los temerarios sin conocimientos se tornan en locos, mas no son valientes. De tal suerte, los valientes ya no serían los temerarios. Todo este asunto, que se trata de manera muy parecida en Laques 192c ss., está ligado a las nociones de episteme y/o sophía. Si está presente el conocimiento, se da valentía o temeridad, pero si falta, se produce una simple temeridad sin la "belleza" de la virtud entera, de la cual la valentía es parte. Ahora bien, esta conducción socrática de ideas, con tales premisas, provoca el justísimo comentario de Protágoras de que Sócrates cree “...que la valentía y la sabiduría (conocimiento) son lo mismo" (350d).

En dicho contexto donde se busca a toda costa la unidad de las virtudes, Sócrates quiere demostrar que la piedad, la justicia, la moderación y la sabiduría son tan parecidas entre sí que se puede considerar que se trata de la misma virtud. Protágoras contesta (331c): “A mí, Sócrates, no me parece que sea tan sencillo que se pueda estar de acuerdo en que la justicia sea pía y la piedad, justa, sino me parece que hay algo distinto en ello". Ante la insistencia de Sócrates de que las virtudes

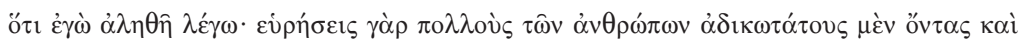

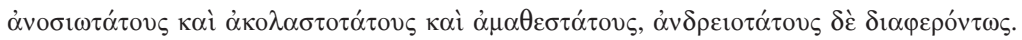


se parecen muchísimo, Protágoras dice, ya molesto (331d): "Pero por supuesto ... la justicia se parece en algo a la piedad, pues cualquier cosa se parece a otra de un modo u otro...", pero $-\mathrm{y}$ esto no lo dice, pero se infiere - , no por ello son lo mismo. Más adelante señala Sócrates (331e): “¿Para ti lo justo y lo pío se comportan de tal modo entre sí que tienen sólo poco de semejante?" Protágoras responde (332a): "No completamente así, pero por cierto no como tú por tu parte pareces opinar". Claramente es Protágoras quien aquí hace el trabajo más fino, detallado y matizado.

Todavía dentro de la supuesta demostración de la unidad de las virtudes, Sócrates usa una "prueba" nada convincente y cuya conclusión no es evidente a primera vista. Se trata del ejemplo de que una cosa sólo puede tener un solo contrario (cf. 332a ss.):

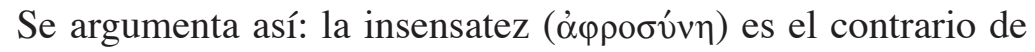
la sabiduría (бopí $\alpha)$. Más adelante, a través de distintos pasos,

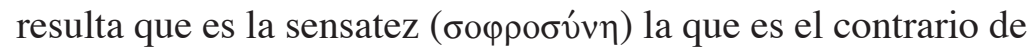

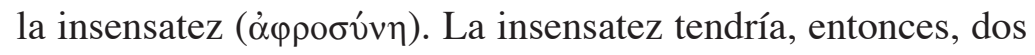
contrarios, a saber, la sabiduría y la sensatez; pero, puesto que se admitió antes que algo sólo puede tener un solo contrario, se infiere que la sensatez y la sabiduría son lo mismo.

En segundo lugar, se da una discusión acerca del placer y del dolor. Sócrates quiere conceder, al menos aparentemente, que lo doloroso (molesto) es malo, y lo placentero, bueno. Dice Protágoras (351 c-d):

No sé, Sócrates, así de sencillo, como tú preguntas, si debo contestar que las cosas placenteras son todas buenas y las molestas, malas; pero me parece no sólo con respecto a la respuesta de ahora que se debe contestar con más exactitud, sino también con respecto a toda mi vida; que hay, por un lado, cosas entre las placenteras que no son buenas, y por otro, a su vez, cosas entre las molestas que no son malas...

Otra vez Protágoras enfatiza que se debería profundizar más en este tema de lo que Sócrates lo hace (en esta ocasión). 
En tercer lugar, en 360a, Protágoras muestra nuevamente mucha sensatez; señala acertadamente que se había admitido una premisa que después, con otro tipo de argumentación, no necesariamente correcta, se vendría abajo. Dice: "Pero si concedemos esto [de que los cobardes no quieren ir hacia lo más bello y placentero], destruimos las concesiones anteriores", [de que lo bello y bueno también es placentero y, por lo tanto, deseable por todos].

Finalmente, quisiera hacer un comentario con respecto a este afán platónico de hacer de las virtudes, especialmente de la valentía, conocimientos. El Protágoras es una de las primeras obras de Platón, en la cual el filósofo prepara ya el camino para hacer de las virtudes un conocimiento, "puesto que sólo conocimientos son enseñables", doctrina eminentemente importante en el pensamiento platónico. En el Menón ya tenemos la virtud como idea innata que debe ser desarrollada mediante la enseñanza. Ello permite que en el mismo Menón se postule un estadista que sepa hacer estadista a otro; esto es, que sepa enseñar la virtud, incluyendo la valentía. Ese es exactamente el programa de la República, del estado ideal y perfecto, una de cuyas condiciones es la enseñabilidad de la virtud, y justo presenciamos los primeros pasos de este camino, no todos logrados, en el diálogo que aquí nos ocupó.

Protágoras muestra un hermoso rasgo de carácter al finalizar la obra (361c), despidiéndose así de Sócrates:

... Sócrates, alabo tu brío y tu modo de conducir los discursos ... a muchos he dicho ... que ... te quiero especialmente ... y digo ... que no me extrañaría si llegaras a estar entre los varones afamados por su sabiduría. 